\title{
Directive excitation of guided electromagnetic waves through polarization control
}

\author{
Jorge Carbonell, ${ }^{1, *}$ Francisco J. Rodríguez-Fortuño, ${ }^{2}$ Ana Díaz-Rubio, ${ }^{1}$ Alejandro Martínez, ${ }^{2}$ \\ Francisco Cervera, ${ }^{1}$ and José Sánchez-Dehesa ${ }^{1, \dagger}$ \\ ${ }^{1}$ Wave Phenomena Group, Department of Electronics Engineering, Universitat Politècnica de València, Camino de Vera, s.n. (Building 7F), \\ ES-46022 Valencia, Spain \\ ${ }^{2}$ Nanophotonics Technology Center, Universitat Politècnica de València, Camino de Vera, s.n., ES-46022 Valencia, Spain \\ (Received 7 November 2013; revised manuscript received 27 March 2014; published 15 April 2014)
}

\begin{abstract}
Experimental evidence is reported on the control of the directionality of guided electromagnetic microwaves by the polarization of the exciting wave. Experiments are conducted using a two-dimensional waveguide made of two parallel aluminum plates. The upper plate, which has a linear array of holes, is externally illuminated by a polarized wave whose incident wavevector is contained within the mirror-symmetry plane defined by the linear array. Surprisingly, the measurements show that the propagation inside the waveguide is highly asymmetrical, and it is controlled by the polarization of the incoming wave. This extraordinary phenomenon is explained in terms of a simple model involving a set of dipoles that are excited at the hole positions. Our finding provides a powerful method to sort different polarizations of a free-space beam to different propagation directions of guided electromagnetic waves.
\end{abstract}

\section{INTRODUCTION}

Excitation of electromagnetic (EM) waves in a controlled direction is a major area of interest due to the large number of potential applications it may have. Although this phenomenon is central to the fields of antennas and lenses, in which directionality of free-space radiated waves is achieved, it also has evident applications in the field of guided propagating waves. Traditionally, in the case of microwave frequencies, the nonreciprocal behavior of magnetized elements has been the basis of guiding waves to the desired spatial path [1]. This interest can be extended to the EM spectrum up to optics, where microstructured media based on subwavelength-size inclusions are key to synthesize artificially designed propagation conditions [2-4]. Within this metamaterial context, for example, chirality has been employed to asymmetrically transmit light with circular polarizations $[5,6]$ or linear polarizations [7-9]. During the last decade, some alternative wave routing concepts have been investigated through the proper design of artificial media, including, for example, active control [10] and extraordinary transmission phenomena [11]. More recently, it has been demonstrated that directionality can be achieved by the near-field interference response of subwavelength elements $[12,13]$. In these cases, the combination of constructive and destructive excitation of subwavelength EM sources permits obtaining unidirectionality of propagating surface plasmons in the optical range.

This paper shows that, at microwave frequencies, it is possible to achieve asymmetric unidirectional excitation of guided EM waves in a mirror-symmetric structure. Moreover, although the incident wavevector is contained within the symmetry plane, it is demonstrated that unidirectional excitation occurs due to the asymmetry of the electric field vector associated with the incident wave. The polarization and the oblique incidence of the excitation wave are responsible

\footnotetext{
*Corresponding author: jorcarol@upvnet.upv.es

†jsdehesa@upv.es
}

for the directionality of the transmitted wave, which is of the transverse electromagnetic (TEM) type, in a two-dimensional (2D) guided medium. As a result, a simple tuning of the incident polarization can be used to completely switch the direction of the excited TEM wave. To show this, we consider two cases of a free-space plane wave illuminating coupling structures. They are based on arrays of subwavelength circular holes or rectangular slits patterned on the top metallic plate of a parallel plate waveguide (PPW) and designed to work with linear or circular incident polarizations, respectively. Then, we provide a simple equivalent model based on aggregated electrical dipoles in the PPW to explain the coupling mechanism and the unidirectional excitation of guided modes. The rationale behind this phenomenon is attributed to the constructive-destructive interference between the dipoles of this model. Numerical and experimental results are analyzed to confirm the feasibility of such a directional propagation approach. Both qualitative and quantitative estimations of such directional behavior are provided.

\section{COUPLING STRUCTURES BASED ON SUBWAVELENGTH APERTURES}

We first analyze a structure that achieves unidirectionality of guided modes for linearly polarized incident plane waves. It consists of a linear array of circular holes on the top metal plate of a parallel plate setup. The hole array behaves as a coupling structure between the external plane wave and the guided (fundamental TEM) wave of the PPW. A linearly polarized plane wave impinges on the linear hole array with incidence angle $\theta$, as depicted in Fig. 1(a). The incoming wave can be decomposed into two linearly polarized waves, $E_{H}$ and $E_{V}$. The contributions of these two waves illuminate the whole array (each successive aperture with an increasing phase delay). The combined response of the aperture array is then coupled to the interplate region of the PPW. The individual contributions of each aperture add in a constructive-destructive scheme as they become secondary radiators. This is an effect similar to the one recently described as generalized Huygens' 
(a)

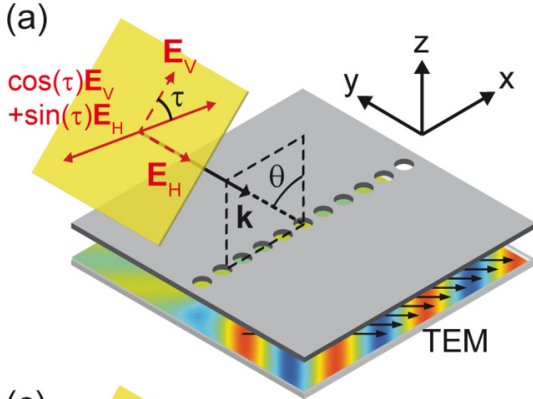

(c)

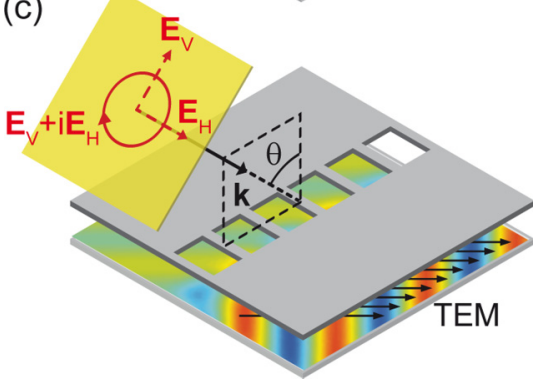

(b)

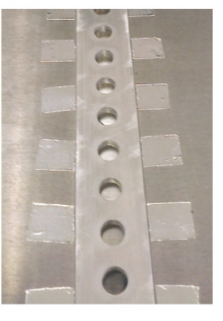

(d)

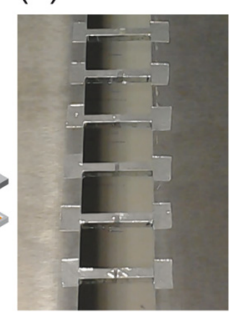

FIG. 1. (Color online) (a) Schematic of a circular hole array with linear incident wave polarization. (b) Manufactured circular array. (c) Schematic of a rectangular slit array with circular incident wave polarization. (d) Manufactured rectangular array.

principle [14], but here we shift the propagation plane perpendicularly. Identical considerations also describe the second structure under analysis, which achieves unidirectionality for circularly polarized incident light and uses an array of rectangular slits instead of one of circular holes [Fig. 1(b)]. The fabricated arrays for experimental validation are shown in Fig. 1(b) and 1(d). In Fig. 1(b), the hole diameter is $\phi=$ $10 \mathrm{~mm}$ and the period is $p_{\text {circ }}=18 \mathrm{~mm}$; in Fig. 1(d), the slit dimensions are $35 \times 26 \mathrm{~mm}$ and the period is $p_{\text {rect }}=31 \mathrm{~mm}$.

\section{DIPOLE MODEL FOR UNIDIRECTIONAL PROPAGATION}

\section{A. Model conception}

To describe the unidirectional excitation of TEM modes inside the PPW, we consider a simple 2D dipole model for the structure depicted in Fig. 1(a) and 1(b). The incident electric field induces opposite charge accumulations in the top plate at opposite ends of each circular hole. This creates, inside the PPW, vertical electric fields of opposite signs that can be modeled with a pair of $z$-oriented electrical dipoles [Fig. 2(b)] placed at either side of each subwavelength hole. The position of these dipoles is determined by the oblique angle $\tau$ of the linearly polarized incident $E$ field, with a separation equal to the diameter of the holes $\phi$. The radiation pattern of such a dipole array in the 2D plane of the PPW can be determined as the product of two patterns. First, the radiation pattern produced by a single hole (modeled by a pair of dipoles) resembles a "figure 8" pattern for subwavelength holes, and shows no unidirectionality [Fig. 2(c)]. Second, we consider the radiation pattern produced by the hole array (array factor). Each hole is excited with a phase shift of $\Delta \phi=k_{0} p \sin (\theta)$ with respect to the previous one, determined by the incident angle of the plane wave, so that only TEM waves propagating
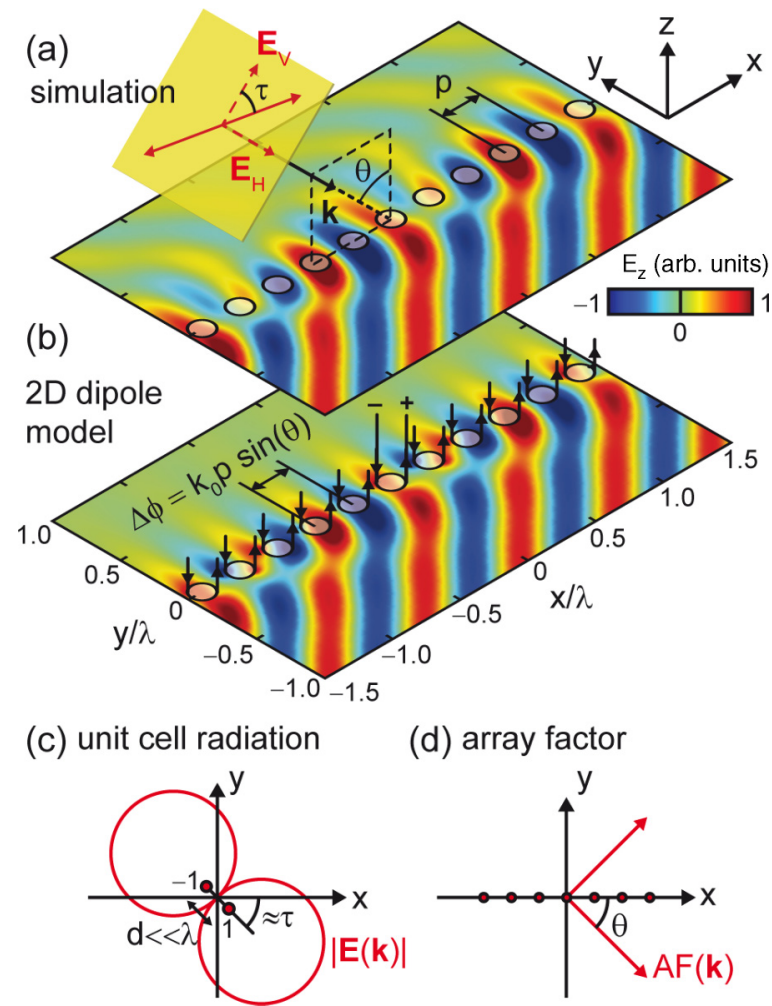

FIG. 2. (Color online) (a) Simulation of the vertical electric field at the bottom plate of the PPW for a linearly polarized plane wave incident at an angle into the array of small circular holes shown in Fig. 1(a). (b) Analytical 2D model of an array of dipoles placed at opposite ends of each hole driven by the incident plane wave. (c) Radiation diagram of TEM waves inside the PPW for each pair of dipoles with opposite signs representing a single hole. (d) Array factor of the periodic repetition of holes are excited by a phase difference of $\Delta \phi$.

at an angle $\pm \theta$ with the $x$ axis are allowed inside the PPW [Fig. 2(d)]. This precludes radiation in one of the lobes of the figure-8 radiation pattern, resulting in unidirectionality. This is shown in the full calculation of the 2D dipole model, greatly resembling the full-wave simulation of Fig. 2(a). By changing the incident linear polarization angle by $90^{\circ}$, we can switch the direction of excitation.

\section{B. Dipole model description}

Sorting of linearly polarized light by means of small subwavelength holes can be explained from a simple 2D model of dipoles. Each hole is modeled by a pair of vertically oriented dipoles with opposite current directions. These dipoles represent the varying accumulation of opposite charges at opposite ends of each hole due to the incident linear polarization. In the 2D model, the dipoles can be treated as infinite oscillating current lines invariant with $z$ so that in a cylindrical coordinate system $(\rho, \phi, z)$, the complex phasor for the electric field radiated by each current line is oriented along $z$ and is given by

$$
E_{z}=-I_{e} \frac{k_{0}^{2}}{4 \omega \varepsilon_{0}} H_{0}^{(1)}\left(k_{0} \rho\right),
$$

where $I_{e}$ is the amplitude of the current per unit length, $\omega$ is the angular frequency, $\varepsilon_{0}$ is the free-space permittivity, and $H_{0}^{(1)}\left(k_{0} \rho\right)$ is the Hankel function of the first kind. The 

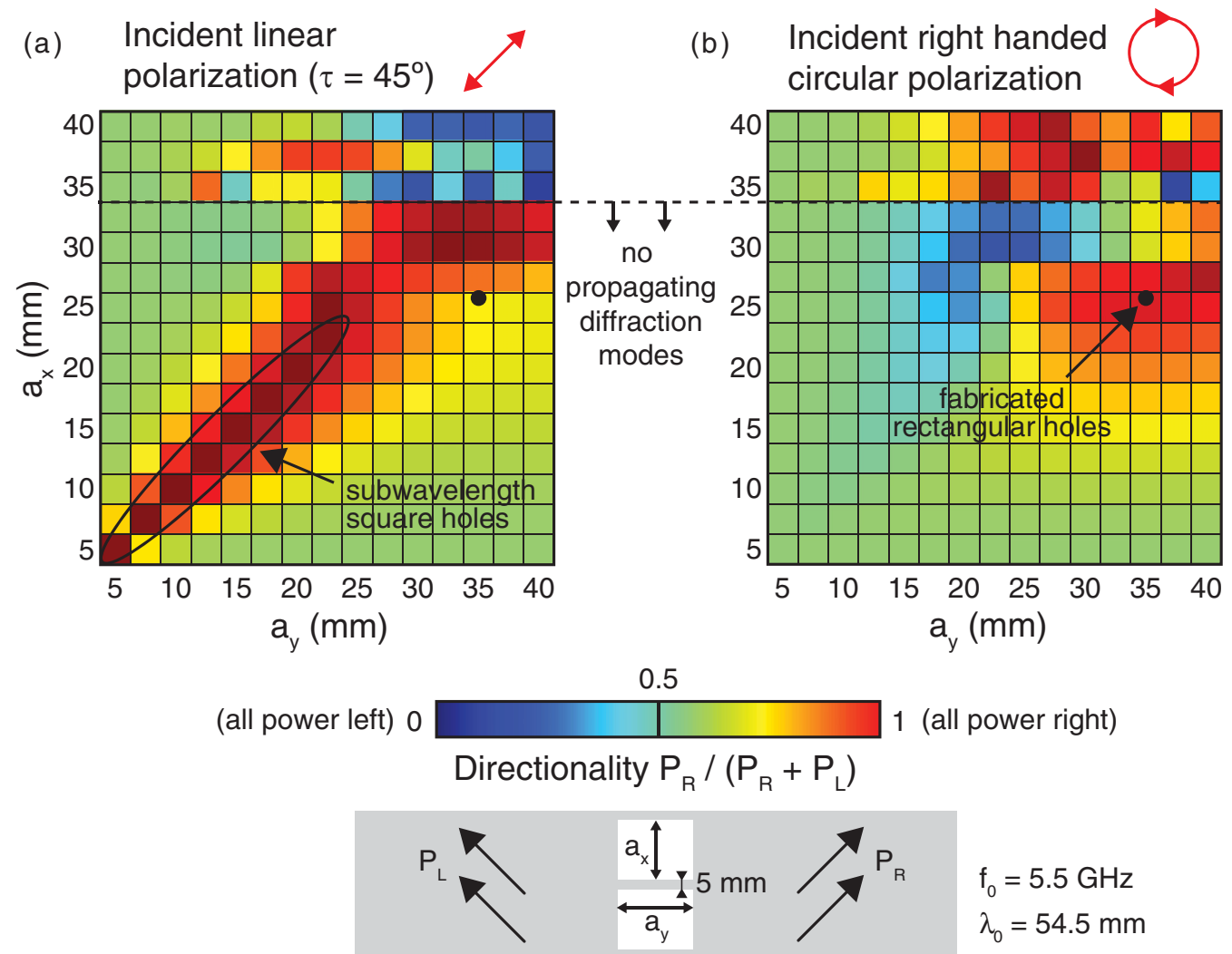

FIG. 3. (Color online) Numerical simulations of the power directionality coefficient $P_{\text {right }} /\left(P_{\text {right }}+P_{\text {left }}\right)$ for the rectangular slits under (a) linear polarization and (b) circular polarization. The selected geometry is marked as a black dot in panels (a) and (b), but it has only been experimentally assessed in the case of circular polarization of panel (b).

coherent addition of this function for each dipole in the model with the appropriate phase in $I_{e}$ leads to the field profile shown in Fig. 2(b).

For a single hole, modeled as two opposite current lines with a subwavelength separation $d$ placed at an angle $\tau^{\prime}(\approx \tau)$ with the $x$ axis, the far field radiation can be expressed as the coherent addition of cylindrical waves generated from two point sources, which, under the parallel ray approximation valid for large distances, is given by

$$
\begin{aligned}
& \left|E(\phi)_{\text {single hole }}\right| \propto\left|1-\exp \left(i k_{0} d \cos \left(\phi+\tau^{\prime}\right)\right)\right| \\
& \quad=2\left|\sin \left(\pi \frac{d}{\lambda} \cos \left(\phi+\tau^{\prime}\right)\right)\right| \\
& \quad \approx\left|\pi \frac{d}{\lambda} \cos \left(\phi+\tau^{\prime}\right)\right| \text { when } d \ll \lambda,
\end{aligned}
$$

which results in the figure-8 pattern shown in Fig. 2(c). The angle of the dipoles given by $\tau^{\prime}$ is approximately equal to the angle of incident linear polarization $\tau$, which induces the charge accumulations on the top plate. This radiation diagram of a single hole still needs to be multiplied by the array factor $A F$ of the periodic array of holes separated a distance $p$ along the $x$ axis, with a constant phase shift of $\Delta \phi=k_{0} p \sin (\theta)$ between elements, determined by the incident plane wave angle of incidence. The array factor for such a linear array is well known and is given by

$$
A F=\frac{\sin (N \psi / 2)}{\sin (\psi / 2)}, \text { with } \psi=k_{0} p \cos (\phi)+k_{0} p \sin (\theta),
$$

which in the limit $N \rightarrow \infty$ (corresponding to an infinite array of holes) tends to the two delta functions $A F \propto$ $\left[\delta\left(\phi-\frac{\pi}{2}+\theta\right)+\delta\left(\phi+\frac{\pi}{2}+\theta\right)\right]$ shown in Fig. 2(d). This results in two allowed propagation directions.

Finally, if the incidence angle and the angle of linear polarization are adjusted so that $\theta=\tau^{\prime}=\pi / 4$, then the array factor selects from the figure- 8 pattern exactly one maximum and one direction of zero radiation, as graphically seen in Fig. 2(c) and (d). Thus, the total radiation obtained by multiplying (2) and (3) shows perfect unidirectionality in the direction $\phi=\pi / 4$, given by $|E(\phi)| \propto|\delta(\phi+\pi / 4)|$.

\section{Dipole model extension}

This model concept remains valid for other shapes of subwavelength-size holes. Thus, different hole shapes can be devised to sort arbitrary polarization states. A limitation can be the appearance of higher-order grating modes, but this can be avoided by using a subwavelength periodicity, as discussed in the Appendix. Hence, a tradeoff exists between a small periodicity to avoid grating effects and a large hole to achieve significant TEM mode excitation.

Regarding our second rectangular structure [Fig. 1(c) and (d)], the use of apertures with a size comparable to the wavelength may involve a complex model with at least two pairs of dipoles per unit cell placed at nonnegligible electrical distances. These conditions include a number of extra effects that add complexity but do not bring essential physical insight, so the dipole model is not adapted to the 

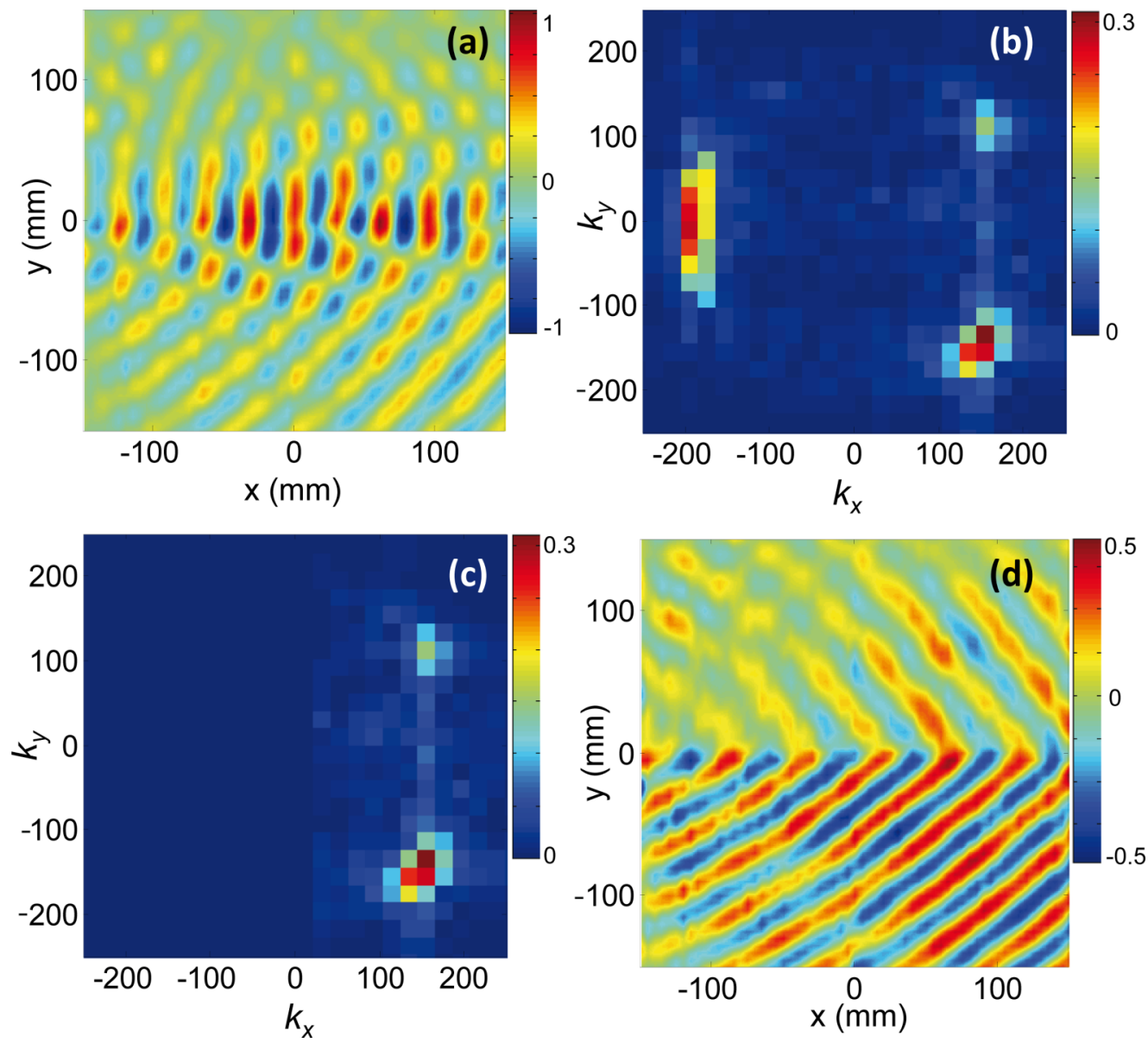

FIG. 4. (Color online) (a) Measured (raw) electric field distribution at $9.3 \mathrm{GHz}$ within the PPW following Fig. 1(a), with the circular hole array located along axis $y=0$. (b) $E$ field processed by means of a FFT. Some backward waves (values with $k_{x}<0$ ) are observed in (b), which are filtered from the $k$ space in (c). (d) Inverse FFT of (c) showing only the forward spectral components of the raw measurement in (a).

rectangular apertures. Instead, we exploit the added complexity to demonstrate the potential for new functionalities. Numerical optimization is performed on the height and width of an array of rectangular slits to achieve unidirectional sorting of linearly polarized waves [Fig. 3(a)] and circularly polarized plane waves [Fig. 3(b)]. In what follows, we will focus our attention to the circular polarization case.

In order to sort different circular polarizations using rectangular holes, we numerically optimized the geometry to achieve a large contrast ratio between left and right TEM $m=$ 0 mode propagation when impinging with circularly polarized fields. Figure 3(b) shows the directionality as a function of the width $\left(a_{y}\right)$ and height $\left(a_{x}\right)$ of the rectangular apertures. The directionality is defined as the fraction of power going to the right for the $m=0$ mode over the total power excited in the two left and right $m=0$ modes. For the rectangular slits, the frequency is invariant, and the metal bridge between successive holes is kept constant at $5 \mathrm{~mm}$. Therefore, the periodicity is a function of $a_{x}$ and has the dependence $p_{\text {rect }}=a_{x}+5 \mathrm{~mm}$. A discontinuity in the directionality is observed for the values of $a_{x}$ at which the condition in Eq. (A3) in the Appendix is no longer fulfilled. This threshold corresponds to the onset of high-order propagating diffraction modes, which can carry power with different directionality.
As can be seen in Fig. 3(b), small rectangular holes do not achieve circular polarization sorting, and larger holes (preferably $>25 \mathrm{~mm}$ in both directions) are required. The simple two-dipole model is not sufficient to describe large slits, so we preferred to use numerical parameter sweeps to evaluate performance. The fabricated array of rectangular holes that achieves good circular polarization sorting directionality is indicated with a black spot in Fig. 3(b).

From a symmetry point of view, the unidirectionality exhibited by both mirror-symmetric structures (circular and rectangular) arises from the asymmetry in the polarization of the free-space excitation. This concept can be compared to the polarization-controlled unidirectionality of optical surface plasmons reported in $[2,12,13]$, but apart from the different frequency regimes, several interesting conceptual differences exist. We apply the phenomenon to both circularly and linearly polarized waves, and we use conventional guided TEM waves, whose wavevectors are inside the light cone (unlike surface plasmons or spoof plasmons). The coupling mechanism is clearly different, and, contrary to $[12,13]$, in our case a single infinite slit does not result in unidirectionality. The dipole model presented is similar to that in Ref. [2]; however, in [2] the unidirectionality is provided by each unit cell under normal incidence, because of the use of two apertures, 

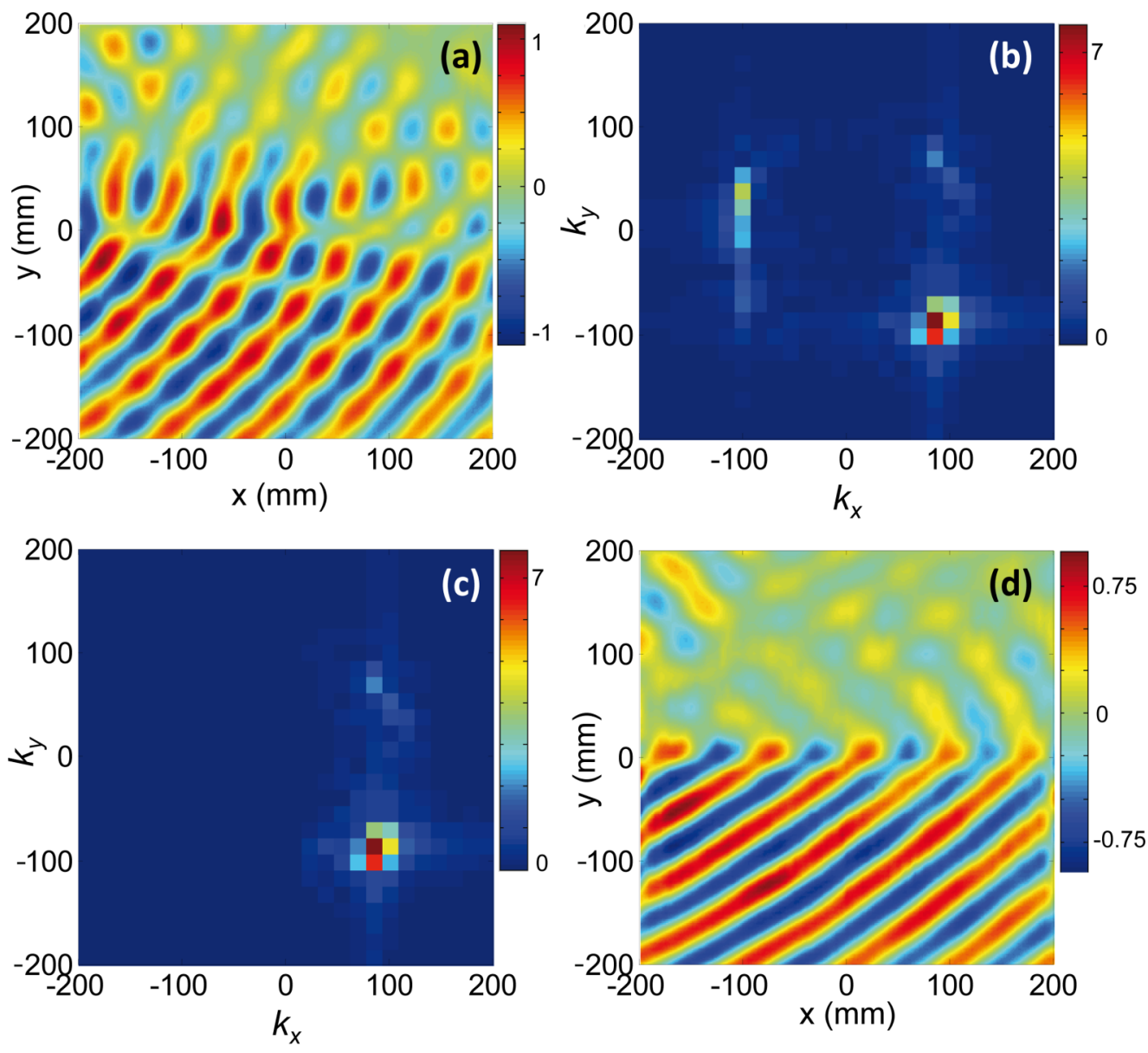

FIG. 5. (Color online) (a) Measured (raw) electric field distribution at $5.5 \mathrm{GHz}$ within the PPW following Fig. 1(c), with the rectangular slit array located along axis $y=0$. (b) $E$ field processed by means of a FFT. Some backward waves are observed in (b), which are eliminated from the $k$ space in (c). (d) Inverse FFT of (c) showing only the forward spectral components of the raw measurement in (a).

modeled as two radiators excited with different phase and orientation. In our case, we use only one aperture per unit cell (a simpler design at the cost of less flexibility), and the nonnormal incidence is an essential ingredient for the unidirectionality. Similar to Refs. [12,13] but contrary to [2], our structure has a double mirror symmetry, meaning that, under normal incidence, the different incident polarizations (e.g., $\tau=\pi / 4$ and $\tau=-\pi / 4$ ) would formally admit the same mathematical description under a reflection of the $x$ axis. Thus, no unidirectionality can exist at normal incidence.

\section{EXPERIMENTAL RESULTS}

\section{A. $E$-field mappings}

To demonstrate our ideas, we have implemented an experimental setup based on a previously reported mapping system [15], where the complex $E$ field is mapped in the PPW near the coupling elements arrays. The experiment is carried out at microwave frequencies, although scalability to higher frequencies should apply. In practice, a nominal $9.3-\mathrm{GHz}$ linearly polarized (and tilted $\tau=\pi / 4$ ) wave is employed with the circular holes array, and a nominal $5.5-\mathrm{GHz}$ circularly (right-handed) polarized wave is used with the rectangular slits array. In both cases, incidence angle is $=\pi / 4$. Importantly, the electrical sizes of the corresponding periodicities $p_{\text {rect }}$ and $p_{\text {circ }}$ are similar, equivalent to $p_{\text {rect }} / \lambda=0.57$ and $p_{\text {circ }} / \lambda=$ 0.56 , respectively. Measurements of the vertical $E$ field $\left(E_{z}\right.$ component) are displayed in Figs. 4 and 5 for the circular holes array and the rectangular slits array, respectively. Figs. 4(a) and 5(a) show the raw measurements obtained for the real part of $E_{z}$ from the network analyzer. In both cases, the incident wave comes from the left and travels toward the $+x$ direction. Even if some unwanted effects are superposed to this raw measurement, it is possible to clearly observe higher field intensity toward the right-hand side of the aperture arrays. A plane wave front is generated at an angle with respect to the array equal to that of the incident wave $\theta$. This excited wave is asymmetrical with respect to the array axis; therefore, unidirectionality is obtained. To understand the exact information contained in this field map, a 2D fast Fourier transform (FFT) is applied to the complex $E$ field, which is displayed in Figs. 4(b) and 5(b). There, two main $k$ components can be identified. In the positive $k_{x}$ quadrants, the unidirectional wave initially expected is clearly identified. Both $k_{x}$ and $k_{y}$ components of the $k$ vector have equal values and therefore confirm the $\pi / 4$ directionality. The "spots" with $k_{x}<0$ in the $k$ space correspond either to higher diffraction order waves, caused by the periodicity of the arrays, or to reflections from the experimental setup. We can verify the basic relation $k_{x}^{2}+k_{y}^{2}=\frac{\omega^{2}}{\mathrm{c}^{2}}$ from an experimental point 
TABLE I. Measured and theoretical values from the $E$-field plots of Figs. 3 and 4 for the $k$ vector, directional angle, and equivalent frequency.

\begin{tabular}{lccccc}
\hline \hline & $\begin{array}{c}k_{x} \\
(\mathrm{rad} / \mathrm{m})\end{array}$ & $\begin{array}{c}k_{y} \\
(\mathrm{rad} / \mathrm{m})\end{array}$ & $\begin{array}{c}k_{0} \\
(\mathrm{rad} / \mathrm{m})\end{array}$ & $\begin{array}{c}\text { Angle } \theta \\
(\mathrm{deg})\end{array}$ & $\begin{array}{c}f \\
(\mathrm{GHz})\end{array}$ \\
\hline $\begin{array}{l}\text { Rectangular Slits } \\
\text { Measured }\end{array}$ & 85.33 & -85.33 & 120.67 & $45^{\circ}$ & 5.76 \\
$\begin{array}{l}\text { Theory } \\
\text { Circular Holes }\end{array}$ & 81.45 & -81.45 & 115.19 & $45^{\circ}$ & 5.5 \\
$\begin{array}{l}\text { Measured } \\
\text { Theory }\end{array}$ & 154.5 & -133.9 & 204.45 & $49.1^{\circ}$ & 9.76 \\
\hline \hline
\end{tabular}

of view with good accuracy, even though some deviation arises due to the limited spatial resolution of the measured data.

A summary of the collected data can be found in Table I, together with the expected theoretical values. As previously stated, periodicity was taken to be as large as possible but trying to avoid the propagation of grating modes. At the nominal frequencies, the first grating modes are under cutoff conditions in both experiments (see the Appendix). Still, because the operation frequencies are close to the cutoff ones, there is a nonnegligible regressive contribution appearing in the $k$ space. In order to discard this unwanted effect, a filtering function is applied in the $k$ space by canceling this regressive wave component. The result of applying a $k_{x}>0$ filter is displayed in Figs. 4(c) and 5(c). Finally, if we perform an inverse FFT on these processed measurements, the progressive contributions of the $E$ field can be restored without the interfering modes. A "cleaned" $E$-field map is obtained in Figs. 4(d) and 5(d), clearly demonstrating that unidirectionality is the fundamental response of the slit and hole arrays to the polarized waves.

\section{B. Quantitative assessment}

Though Figs. 4 and 5 give qualitative assessment of the unidirectionality phenomenon as observed from the $E$-field patterns, quantitative evaluation can be provided by further analyzing the measured data. As we are interested in a relative figure of merit to measure how much unidirectionality is obtained, we evaluate the unbalanced power transmission to each side of the aperture arrays as a power ratio of the integral of the squared $E$ field at each side of the array axis:

$$
P_{\text {right }} / P_{\text {total }} \propto\left|E_{\text {right }}^{z}\right|^{2} /\left(\left|E_{\text {right }}^{z}\right|^{2}+\left|E_{\text {left }}^{z}\right|^{2}\right)
$$

Results for both arrays are plotted in Fig. 6. Numerical data have been included from finite element simulations (with High Frequency Structure Simulator from Ansys) of both structures, and by using the corresponding frequency and polarization. To avoid misleading values for measured and simulated data, the areas corresponding to the surface exactly under the aperture arrays are excluded from the calculation, since they contain significant evanescent field components. Hence, only the areas that have the guided plane wave fields toward each side are considered. This calculation is also performed on the filtered data of Figs. 4(d) and 5(d). An important conclusion from this result is that unidirectionality is a broadband phenomenon, since a high ratio is obtained in a wide frequency band. This is because

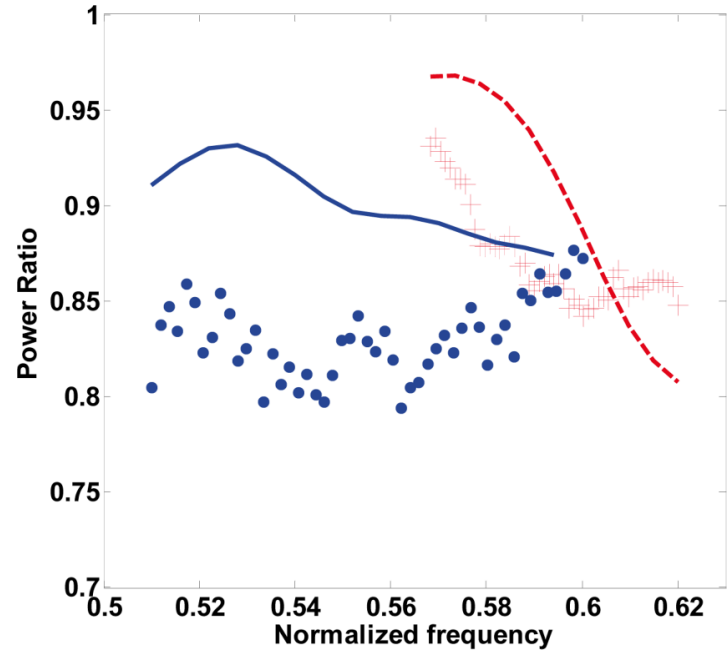

FIG. 6. (Color online) Measured (symbols) and simulated (lines) unidirectionality coefficients as a function of the normalized frequency $f_{\text {norm }}=f p_{\text {array }} / c$ for the hole (blue solid) and slit (red dashed) coupling structures under linear and circular polarizations, respectively.

subwavelength (i.e., nonresonant) elements are employed. We have employed a variable frequency because it is instantaneous to perform frequency sweeps from the experimental point of view. The observed ratio slightly decreases with frequency because the apertures become electrically larger and grating effects due to their periodicity occur. The comparison shows good agreement between the measured and the simulated results. We may attribute the differences, smaller than $10 \%$, to imperfections in the measurement setup caused by internal reflections in the PPW.

\section{CONCLUSIONS}

We have demonstrated that unidirectionality of guided waves can be achieved by controlling the polarization of the excitation wave. This behavior applies to circular and linear polarizations and is driven by the incidence angle of the excitation wave. Simple coupling structures may be used with circular or rectangular shapes to force transmission of the guided wave in a quasiunidirectional way. A simple dipole-based model explains the observed behavior through the constructive-destructive interference among the different contributions of the response of the apertures. This phenomenon can be used to design devices acting as polarization duplexers, i.e., devices that can separate different signals incoming to a receiver based on their orthogonal polarizations. In addition, it may add information about the relative angle between emitter and receiver.

\section{ACKNOWLEDGMENTS}

The authors gratefully acknowledge B. Bernardo and A. Vila at Electromagnetic Radiation Group Universitat Politècnica de València for the generous donation of the circular polarization antenna for the experiments. J.S.-D. thanks D. Torrent for useful discussions. Financial support from the Spanish Ministry of Economy and Competitiveness (Grants No. TEC2010-19751, No. TEC2011-28664-C02, and No. Consolider CSD2008-00066) is also acknowledged. 


\section{APPENDIX: DIFFRACTION GRATING EFFECTS DUE TO PERIODICITY}

In order to understand the propagation direction of the TEM modes inside the PPW, we have considered the following conditions:

(1) The TEM modes inside the PPW must follow the 2D wave equation; therefore, their propagating wavevectors must fulfill the following condition:

$$
k_{x}^{2}+k_{y}^{2}=k_{0}^{2}
$$

(2) The modes inside the PPW are excited by the diffraction of the incoming plane wave in the hole array. The wavevector component $k_{y}$ is not conserved due to diffraction occurring on a single element along $y$, but the wavevector component $k_{x}$ must fulfill the requirement imposed by the periodicity of the hole array oriented along $x$, given by

$$
k_{x}=k_{x}^{\text {inc }}+m \frac{2 \pi}{p},
$$

where $k_{x}^{\text {inc }}=k_{0} \sin (\theta)$ is the wavevector $x$ component of the incident plane wave, $p$ is the periodicity of the array, and $m$ is an integer, which defines the different diffraction orders.

Combining Eqs. (A1) and (A2), we obtain the possible directions of the modes inside the PPW, which also depend on the operating frequency and the periodicity. A graphical solution to the two conditions is depicted in Fig. 7. The zero-order mode has $k_{x}^{m=0}=k_{x}^{\text {inc }}=k_{0} \sin (\theta)$ and can exist with either positive or negative $k_{y}$, corresponding to the two propagation directions (left or right). The ratio of the excitation of these two possible directions of the $m=0$ mode is the subject of study of the present paper.

The existence of high-order propagating modes inside the PPW is undesirable for our concept demonstration, so we have to design the periodicity $p$ such that the additional modes are outside the propagating circle, i.e., $k_{x}^{m \neq 0}>k_{0}$. Following

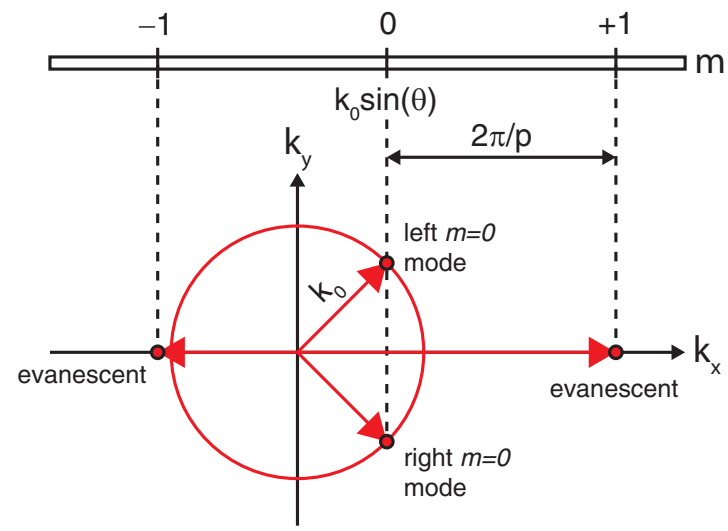

FIG. 7. (Color online) Spatial Fourier space for the guided modes inside the PPW.

Eq. (A1), this means that $k_{x}^{m \neq 0}$ should be imaginary; therefore, the mode amplitudes fade away from the hole array. From Fig. 7, we see that it is sufficient to fulfill this condition for the mode $m=-1$. This requires $k_{0} \sin (\theta)-\frac{2 \pi}{p}<-k_{0}$, which, knowing that $k_{0}=2 \pi / \lambda$, is equivalent to

$$
p=<\frac{\lambda}{1+\sin (\theta)},
$$

which is fulfilled in both examples presented in the paper. Therefore, only the modes with $m=0$ can propagate inside the PPW. However, because the periodicity is just below the required limit, the mode $m=-1$ is close to cutoff; therefore, its exponential decay away from the hole array is relatively slow and affects the measured field profiles near the holes. Also, the finite length of the array relaxes the condition Eq. (A2) by broadening the spatial frequency linewidth of the different grating modes so that the tails of the $m=-1$ mode can enter inside the propagating circle.
[1] R. E. Collin, Foundations for Microwave Engineering (McGraw-Hill, New York, 1992).

[2] J. Lin, J. P. B. Mueller, Q. Wang, G. Yuan, N. Antoniou, X. C. Yuan, and F. Capasso, Science 340, 331 (2013).

[3] L. Huang, X. Chen, B. Bai, Q. Tan, G. Jin, T. Zentgraf, and S. Zhang, Light Sci. Appl. 2, e70 (2013).

[4] B. Tsema, Y. Tsema, M. Shcherbakov, Y. Lin, D. Liu, V. Klimov, A. Fedyanin, and D. Tsai, Optic Express 20, 10538 (2012).

[5] A. Drezet, C. Genet, J. Laluet, and T. Ebbesen, Optic Express 16, 12559 (2008).

[6] A. S. Schwanecke, V. A. Fedotov, V. V. Khardikov, S. L. Prosvirnin, Y. Chen, and N. I. Zheludev, Nano Lett. 8, 2940 (2008).

[7] C. Menzel, C. Helgert, C. Rockstuhl, E. B. Kley, A. Tunnermann, T. Pertsch, and F. Lederer, Phys. Rev. Lett. 104, 253902 (2010).
[8] M. Kang, J. Chen, H. Cui, Y. Li, and H. Wang, Optic Express 19, 8347 (2011).

[9] M. Mutlu, A. E. Akosman, A. E. Serebryannikov, and E. Ozbay, Phys. Rev. Lett. 108, 213905 (2012).

[10] S. Lee, M. Choi, T. Kim, M. Liu, X. Yin, H. Choi, C. Choi, S. Choi, X. Zhang, and B. Min, Nat. Mater. 11, 936 (2012).

[11] M. Beruete, M. Navarro Cia, and M. Sorolla, New J. Phys. 12, 063037 (2010).

[12] F. Rodríguez Fortuño, G. Marino, P. Ginzburg, D. O'Connor, A Martínez, G. Wurtz, and A. Zayats, Science 340, 328 (2013).

[13] S. Y. Lee, I. M. Lee, J. Park, S. Oh, W. Lee, K. Y. Kim, and B. Lee, Phys. Rev. Lett. 108, 213907 (2012).

[14] C. Pfeiffer and A. Grbic, Phys. Rev. Lett. 110, 197401 (2013).

[15] J. Carbonell, A. Díaz-Rubio, D. Torrent, F. Cervera, M. A Kirleis, A. Pique, and J. Sánchez-Dehesa, Sci. Rep. 2, 558 (2012). 